\title{
Optimal Power Allocations of Different Modulations for Multi Channel Cognitive Radio Systems Using Stackelberg Game
}

\author{
Mohammad Obaidur Rahman and Asaduzzaman \\ Department of Computer Science and Engineering \\ Chittagong University of Engineering \& Technology, Chittagong-4349, \\ Bangladesh \\ obaidur_91@cuet.ac.bd and asad@cuet.ac.bd
}

\begin{abstract}
A non-orthogonal multiple access scheme e.g. CDMA based cellular multi-channel cognitive radio network (CRN) is applied in our scheme. Here multiple secondary users share a common set of channels simultaneously. For such an environment, we develop a distributed power allocation approach based on a game theoretic formulation among secondary users to satisfy their rate requirement. We model the problem using a twostage leader-follower game known as Stackelberg game where base station and users are considered as leader and followers respectively. Since the spectrum is licensed to a primary network, base stations of the cellular secondary network should protect primary users against interference caused by secondary communications. We are interested for downlink power allocation with pricing that maximizes utilities of both BS and CRs in presence of primary network. A nonlinear relationship between bit rate and SINR which is solved by game theory. Simulation results show that our proposed power control scheme provides higher SINR levels (or equivalently higher data rates) while consuming lower average transmit power of secondary users. We illustrate that the solution from the distributed formulation conforms the centralized solution. We also compare the above results for different spectrum usage of QAM, ASK and PSK modulation schemes. Moderate spectrum usages give better result.
\end{abstract}

Keywords: Cognitive radio network(CRN), power allocation, non-cooperative game, Stackelberg game, Nash equilibrium(NE), Stackelberg equilibrium(SE), pricing, QAM, $A S K, P S K$ and BER

\section{Introduction}

Power control of users in a cognitive radio has been a important topic for wireless communication research over the past several years; it has been analyzed by many distinguished minds and in various well-publicized works. Although in cognitive radio theory the interference control approach can be used in both centralized or distributed wireless networks, this spectrum access paradigm would be more applicable for networks with infrastructure such as cellular networks where channel state information can be readily obtained. In cellular systems the secondary users can be players. The resource allocation should be done in a way that guarantees protection of primary network data communications against excessive interference. In this paper, we focus on the problem of power allocation in the downlink of a cellular cognitive radio network using Stackelberg game theory. We are interested in developing a decentralized scheme for downlink power allocation with pricing that maximizes utilities of both BS and CRs in presence of primary network. Each BS wants to earn higher income from the CRs in its cell while spending

Received (August 5, 2017), Review Result (September 26, 2017), Accepted (October 12, 2017) 
less transmit power. Each $\mathrm{CR}$ is interested in gaining higher level of SINR (or equivalently data rate) with high QoS(BER) while paying less to the associated BS.

\section{Related Work}

Earlier power allocation solutions are Calculas based approach, Convex Optimization, Karush-Khun-Tucker solution, Variational-inequality etc [1]. In [3] the author proposed two stage optimization of power and bit rate sequentially and later simultaneously. They considered all constraints as convex along with objective function that made them convex optimization. Linear pricing game falls in group of Karush-Khun-Tucker solution proposed in [4]. Here cost function is introduced as the difference between the price of transmission power and data rates. But every of them is of single modulation scheme only.

Conversions from convex optimizations to competitive games are highlighted widely at present. One of the methods is done in [5]. They developed game theory based distributed approach to solve power allocation. The solution that is used for game theoretic implementations is the Nash Equilibrium (NE). At a NE point, given the power and rate levels of other uses, no user can improve its utility level by making individual changes in its power and rate. The NE concept results in a stable solution of a game where players with conflicting interests compete through self optimization and reach a point where no player wishes to deviate. If there is a solution to the above game, Then it would be the one that reaches NE [7].

On the other hand, Stackelberg duopoly, also called Stackelberg competition, is a model of imperfect competition based on a non-comparative name. It is a sequential game (not simultaneous as in Cournot's model) [8]. It should be noted that there are few papers in the literature that study the resource management problems using Stackelberg game theory [5]. They are interested in distributed relay selection and power control for multiuser cooperative communication networks.

Previous works on the downlink resource allocation such as consider BSs as players of the game and do not allow CRs to take part in the game [9]. As a result, such approaches do not provide any degree of freedom to CRs in determining there parameters such as requested SINR level, rate, BER etc. However, in this paper we consider both BS and CRs as players in the resource competition game. In such scenario Stackelbergh game is suitable because the BS as a leader and the CRs act as followers.

In [13] to increase spectral efficiency, multiple SUs may coexist in the same channel. Also, channels may be of different quality. Therefore, SUs assigned to higher quality channels may hold an advantage over SUs assigned to the poorer channels and the rate requirement of some secondary users may not be satisfied by allocating one channel to a user. We develop a relationship between bit rate and SINR for a specific BER which is nonlinear. We cannot use Karush-Khun-Tucker conditions to solve the problem. Along with other equation this nonlinear relationship form a game. We use "Interior Point Method" to solve this problem. The main contribution of this paper is to develop utility functions for both BS and CRs, and to formulate a Stackelberg game that maximize the utility functions in presence of linear and nonlinear distributed constraints. Results show that the proposed game provides near optimal rate and power allocation. For multichannel environment all channels are used by all users are expected. But in wireless environment this is rarely present. So, we have prepared spectrum usage pattern by selecting channels by applying in previous all users and all channels condition [13]. Then we compare the above results for different spectrum usage of QAM, ASK and PSK modulation schemes. Several test cases are formed by applying different thresholds of SINR on fully used spectrum case. Moderate spectrum usages give better result. 


\section{Proposed Scheme}

\section{A. Stackelberg Game}

The Stackelberg leadership game model is a strategic game in which there are two types of players; leaders and followers. The leader player moves first and then the follower player moves sequentially. Therefore, the follower chooses a strategy to optimize its own objective function, knowing the leaders move [2]. The leader, thus, has to optimize its own objective function by predicting the optimal response of the follower. As in our power allocation problem CRs choose their requested SINR based on the unitprices previously announced by BSs, we conclude that the strategies of CRs depend on strategies of BSs that move first. Consequently, the Stackelberg game is quite suitable for modeling our problem [9].

\section{B. System Model}

We consider non-orthogonal multiple access scheme e.g. CDMA applied to a underlay cognitive radio network with a total of $\mathrm{M}$ secondary users and $\mathrm{L}$ channels in a typical cell. There are some assumptions about system model, (1) each channel can be used simultaneously by multiple secondary users via some form CDMA access scheme; (2) a single secondary user can use multiple channels at the same time to meet their rate requirements; (3) every active SU radio has an upper limit on power and rate (bits/channel use) at which it can receive; (4) all SUs employ M-ary quadrature amplitude modulation (QAM), amplitude shift keying (ASK) and phase shift keying (PSK) scheme with an adaptable modulation order $\mathrm{M}$ each; (5) simple path loss model for channel has been assumed; (6) each user has a minimum rate and BER constraint that needs to be maintained and (7) an interference temperature threshold to protect possible primary user transmission on any channel.

We model the power allocation problem with non-cooperative two-stage Stackelberg game where BSs as leaders and CRs as followers [9]. The strategy of each BS is to determine the unit-price for each of its CRs and strategy of each CR is to determine the expected SINR level. Each BS tends to earn more utility from the CRs in its cell while spending less transmit power to them. Each CR is interested in gaining higher SINR level (or equivalently data rate) which paying less to the BS of its cell. As a result, the utility functions of BSs and CRs can be defined as follows.

$$
U_{B S}=\sum_{k=1}^{L} \sum_{i=1}^{N_{S}(k)} \Pi_{i} \gamma_{i}(k)-\sum_{k=1}^{L} \sum_{i=1}^{N_{S}(k)} p_{i}(k)
$$

Where $\Pi_{i}$ is the unit-price determined by $B S$ for charging $C R_{i}$ and $\gamma_{i}$ is level of provided received SINR for which is a function of $p$ (the vector of powers). The utility function of each CR can be written as:

$$
\begin{aligned}
& U_{C R j}=\sum_{k=1}^{L} b_{i}(k)-\Pi_{i} \sum_{k=1}^{L} \gamma_{i}(k) \\
& b_{\boldsymbol{i}}(k)=\log \left(+\gamma_{\boldsymbol{i}}(k)\right) \text { and } \gamma_{i}(k)=\frac{p_{i}(k) h_{i, i}(k)}{\sum_{j=1, j \neq i}^{N_{S}(k)} P_{j}(k) h_{j, i}(k) \rho_{j i}^{2}+\sigma^{2}(k)}, \forall i, k,
\end{aligned}
$$


Table 1. Notations

\begin{tabular}{c|c}
\hline \hline$N_{s}(k)$ & Predicted number of users for $k$-th channel \\
\hline$\sigma^{2}(k)$ & Noise variance in $k$-th channel \\
\hline$\rho_{j, i}$ & Orthogonality factor between users $j$ and $i$ \\
\hline$h_{i, i}(k)$ & Power gain from $i$-th transmitter to $i$-th receiver in $k$-th channel \\
\hline$h_{i, m}(k)$ & Power gain from $i$-th transmitter at location m in $k$-th channel \\
\hline$p_{i}(k)$ & Transmit power per bit of $i$-th user in $k$-th channel \\
\hline$p_{i}{ }^{(k)}$ & Interference temperature constraint in $k$-th challel \\
\hline$I_{t h}{ }^{(k)}$ & Rate of $i$-th user in $k$-th channel \\
\hline$b_{i}(k)$ & Maximum rate of $i$-th user in $k$-th channel \\
\hline$b_{i}^{m^{m a x}}(k)$ & Maximum rate supported by $k$-th channel \\
\hline$R_{c h}^{u}(k)$ & Minimum required rate for $i$-th user \\
\hline$R_{i}^{l}$ & BER for $i$-th user in $k$-th channel \\
\hline$p_{e, i}(k)$ & BER threshold at receiver for $i$-th user in any channel \\
\hline$p_{e, i}^{t h}$ & -th user in $k$-th channel \\
\hline \hline
\end{tabular}

\section{Resource Allocation Framework}

The objectives of the optimization framework are (1) to minimize the total transmit power (2) to maximize the total rate. Thus the objective function of both BS and CR users become minimized.

$$
\begin{aligned}
& \text { Determine } \quad\left[P^{T} b^{T}\right]^{T} \\
& \text { where, } p=\left[p_{1}(1) \ldots P_{N_{S}(1)}(1) \ldots P_{N_{S}(L)}(L)\right]^{T} \text { and } \\
& b=\left[b_{1}(1) \ldots b_{N_{S}(1)}(1) \ldots b_{N_{S}(L)}(L)\right]^{T} \\
& \text { To Minimize: } \quad F_{1}=\sum_{k=1}^{L} \sum_{i=1}^{N_{S}(k)} p_{i}(k) \text { and_Maximize: } \quad F_{2}=\sum_{k=1}^{L} \sum_{i=1}^{N_{S}(k)} b_{i}(k) \\
& \text { subject to } \\
& C 1: 0 \leq p_{i}(k) \leq p_{i}^{\max }(k), \forall i, k \\
& C 2: b_{i}(k) \in\left[1, \ldots, b_{i}^{\max }(k)\right], \forall i, k \\
& \text { C3: } \sum_{i=1}^{N_{S}(k)} p_{i}(k) h_{i, m}(k) \leq I_{t h}(k), \forall k \\
& C 4: \sum_{i=1}^{N_{S}(k)} b_{i}(k) \leq R_{c h}^{u}(k), \forall k \\
& C 5: \sum_{k=1}^{L} b_{i}(k) \geq R_{i}^{1}, \forall i \\
& C 6: p_{e, i}(k) \leq P_{e, i}^{t h}, \forall i, k,
\end{aligned}
$$


where, for QAM

$P_{e, i}(k) \leq \frac{4}{b_{i}(k)} Q\left(\sqrt{\frac{3 b_{i}(k) \gamma_{i}(k)}{\left(2^{b_{i}(k)}-1\right.}}\right), \forall i, k$, odd $b_{i}(k) ;$
$P_{e, i}(k)=\frac{4}{b_{i}(k)}\left(1-2^{-\frac{b_{i}(k)}{2}}\right) Q\left(\sqrt{\frac{3 b_{i}(k) \gamma_{i}(k)}{\left(2^{b_{i}(k)}-1\right.}}\right), \forall i, k$, even $b_{i}(k) ;$

for ASK

$P_{e, i}(k)=\frac{2}{b_{i}(k)} Q\left(\sqrt{\frac{6 b_{i}(k) \gamma_{i}(k)}{\left(2^{2 b_{i}(k)}-1\right.}}\right), \forall i, k ;$

for PSK

$$
P_{e, i}(k)=\frac{2}{b_{i}(k)} Q\left(\sqrt{\frac{2 \pi^{2} b_{i}(k) \gamma_{i}(k)}{2 b_{i}(k)}}\right), \forall i, k ;
$$

Here, constraints $\mathrm{C} 1$ and $\mathrm{C} 2$ indicate limits on transmit power and rate, respectively, $\mathrm{C} 3$ indicates the interference temperature constraint: $\mathrm{C} 4$ indicates the total rate supported by a channel: $\mathrm{C} 5$ represents the required rate of users and finally $\mathrm{C} 6$ is BER constraint: $Q(x)$ is defined as $f_{x}^{\infty} e^{-\varsigma^{2} / 2} d \varsigma$. Since $b_{i}(k)$ is discrete and constraint C6 is nonlinear, the optimization formulation presented above is a constrained multi-objective mixed integer nonlinear programming (multiobjecive MINLP) optimization problem, which is NP-hard in general. Relaxing the integer constraint on rate, $b_{i}(k)$ (as assumed in [11]) and assuming $b_{i}(k)$ as continuous variable, the above optimization problem can be restated with $\mathrm{C} 2$ as:

$C 2: 1 \leq b_{i}(k) \leq b_{i}^{\max }(k), \forall i, k$.

As in [1], constraint C6 can be written as

$$
\text { C7 : } \quad-\gamma_{i}(k) \leq-C_{q \text { arg }}\left(2^{b_{i}(k)}-1\right), \forall i, k \text {; }
$$

where, $C_{q \text { arg }}$ is a constant and is also determined following the analysis in [3]. The

resource allocation scheme with combined single objective can be rewritten as:

Minimize $\quad \tau_{1} F_{1}-\tau_{2} F_{2}$

Subject to

$C 1, C 2, C 3, C 4, C 5, C 7$

The parameters $\tau_{1}$ and $\tau_{2}$ in the combined objective function are the scalarization factors and can be set following the discussion in [12].

It is important to note that we can use the solution (Eq. (8)) as a starting point to search for the optimal discrete valued $b_{i}(k)$. That is, we search in the neighborhood of the continuous solution (which serves as the lower bound for the objective in (Eq.(8)) to find the optimal discrete solution $\left(b^{o p t}\right)$. Based on the new discrete solution, the optimal transmit power $\left(p^{o p t}\right)$ is recalculated using (Eq.(8)).

In the next section, we develop a game theoretic solution concept to solve the resource allocation framework. 


\section{Solution Concepts and Distributed Algorithm}

It is easy to verify that the power allocation played by $S U$ s and $B S$ is a potential game with potential function in (1) and (2) which they try to maximize,

$$
\begin{aligned}
& \text { e.g. } \\
& \mathrm{f}(\mathrm{x})=\sum_{k=1}^{L} \sum_{i=1}^{N_{S}(k)} \Pi_{i} \gamma_{i}(k)-\sum_{k=1}^{L} \sum_{i=1}^{N_{S}(k)} p_{i}(k)
\end{aligned}
$$

The strategy spaces of SUs and the power game of the $B S$ are compact and convex sets and the Potential function $\mathrm{f}$ is continuous. Hence there exists at least one $N E$ for the game for each price $\Pi_{i}$. Also the best response iteration converges to a Nash point.

In proposed Game follower Players are said to play rationally if they try to choose an action that, in conjunction with the other player actions, maximizes their utility function. For ease in presentation, we define the action for follower secondary user $i$ as $y_{i}$ and the union set of all other users actions as $y_{-i}$.

$$
\begin{aligned}
& \text { Here } y_{i}=\left[\begin{array}{cc}
p_{i}^{T} & b_{i}^{T}
\end{array}\right]^{T} \text { where } \\
& p=\left\lfloor p_{1}(1) \ldots P_{N_{S}(1)}(1) \ldots P_{N_{S}(L)}(L)\right\rfloor^{T} \\
& b=\left[b_{1}(1) \ldots b_{N_{S}(1)}(1) \ldots b_{N_{S}(L)}(L)\right]^{T}
\end{aligned}
$$

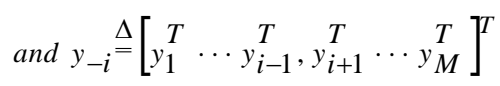

The noncooperative game formulation to determine transmit power and rate can be formally stated as

$$
\begin{aligned}
& \text { Determine } \quad y_{i} \\
& \begin{array}{l}
\text { To Maximize } \quad u_{i}\left(y_{i}, y_{-i}\right) \\
\text { subject to } \\
C G 1: 0 \leq p_{i}(k) \leq p_{i}^{g \max }(k), \quad \forall i, k \\
C G 2: 1 \leq b_{i}(k) \leq b_{i}^{g \max }(k), \quad \forall i, k \\
C G 3: \sum_{k=1}^{L} b_{i}(k) \geq R_{i}^{l}, \quad \forall i, \\
C G 4:-\gamma_{i}(k) \leq-C_{q \arg }\left(2^{b_{i}(k)}-1\right), \\
\forall i, k .
\end{array}
\end{aligned}
$$

It is important to note how the system constrains C3 and C4 is the resource allocation framework (9) are maintained in the formulated noncooperative game. We take a conservative approach to satisfy those constraints in the game formulation.

$$
p_{i}^{g \max }(k)=\operatorname{Min}\left(p_{i}^{\max }(k), \text { Upper_bound_p }\right)
$$

and the upper bound obtained from bound corresponds to $I_{t h}(k) /\left(h_{i, m} N_{s}(k)\right)$ for location $\mathrm{m}$. As an example using Table 9

$$
p_{i}^{g \max }(k)=\operatorname{Min}\left(6, I_{t h}(k) /\left(h_{i, m} N_{s}(k)\right)\right)
$$

Similarly, as an example using Table 9

$$
b_{i}^{g \max }(k)=\operatorname{Min}\left(5, R_{c h}^{u}(k) /\left(N_{S}(k)\right)\right)
$$

In summary, based on local information, we formulate a game to determine suboptimal distribution of power and rate that a secondary user has to employ across the channels in order to minimize total power consumption, maximize rate, and maintain QoS (BER). 


\section{E. Analysis of the Heuristic Solution and Games}

I.: Heuristic and Game: Poorer channels (i.e. lesser SINR) are discarded by heuristic method. Game 1 allocates power to all channels for each secondary user.

\section{Channel Allocation from SINR:}

Algorithm 1 Algorithm for Channel Allocation of the game $G$

Stopping counter, $t=1$;

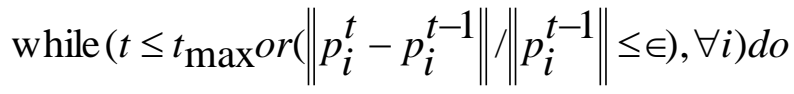

$\%$ Execute optimization problem

for $i=1, \quad 2, \ldots, M d o$

for $k=1,2, \ldots, L \quad d o$

Measure the interference and noise power

$$
\begin{aligned}
& \text { (i.e., } \left.\sum_{j=1, j \neq i}^{N_{S}(k)} p_{j}^{t-1}(k) h_{j, i}(k) \rho_{j, i}^{2}+\sigma^{2}(k)\right) \\
& \text { if } \operatorname{SINR}_{i}(i, k)>\operatorname{SINR}_{\text {threshold }} \quad U(i, k)=1 ;
\end{aligned}
$$

end for

end for

\section{Single Channel Allocation from SINR:}

Algorithm 2 Algorithm for Single Channel Allocation of the game $G$

Stopping counter, $t=1$;

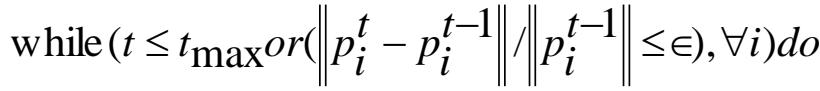

$\%$ Execute optimization problem

for $i=1, \quad 2, \ldots, M d o$

for $k=1,2, \ldots, L \quad d o$

Measure the interference and noise power

$$
\begin{aligned}
& \left(\text { i.e. }, \sum_{j=1, j \neq i}^{N_{S}(k)} p_{j}^{t-1}(k) h_{j, i}(k) \rho_{j, i}{ }^{2}+\sigma^{2}(k)\right) \\
& \text { [sorted_SNR,index] }=\operatorname{sort}(\operatorname{SINR}(1,:)) \\
& U(i, \text { index[end] })=1
\end{aligned}
$$

end for

end for

for $i=1, \quad 2, \ldots, M d o$

for $k=1,2, \ldots, L \quad d o$

if $\quad U(i, k)=1 \quad k k=k k+1$;

end for

end for

if $\mathrm{kk}=0$

for $k=1,2, \ldots, L \quad d o$

[sorted_SNR,index] $=\operatorname{sort}(\operatorname{SINR}(, \mathrm{k}))$

$U($ index $[$ end $], j)=1$

end for

end 


\section{Game: Game Allocates Power to the Selected Channels for each Secondary User.}

\section{Power Allocation:}

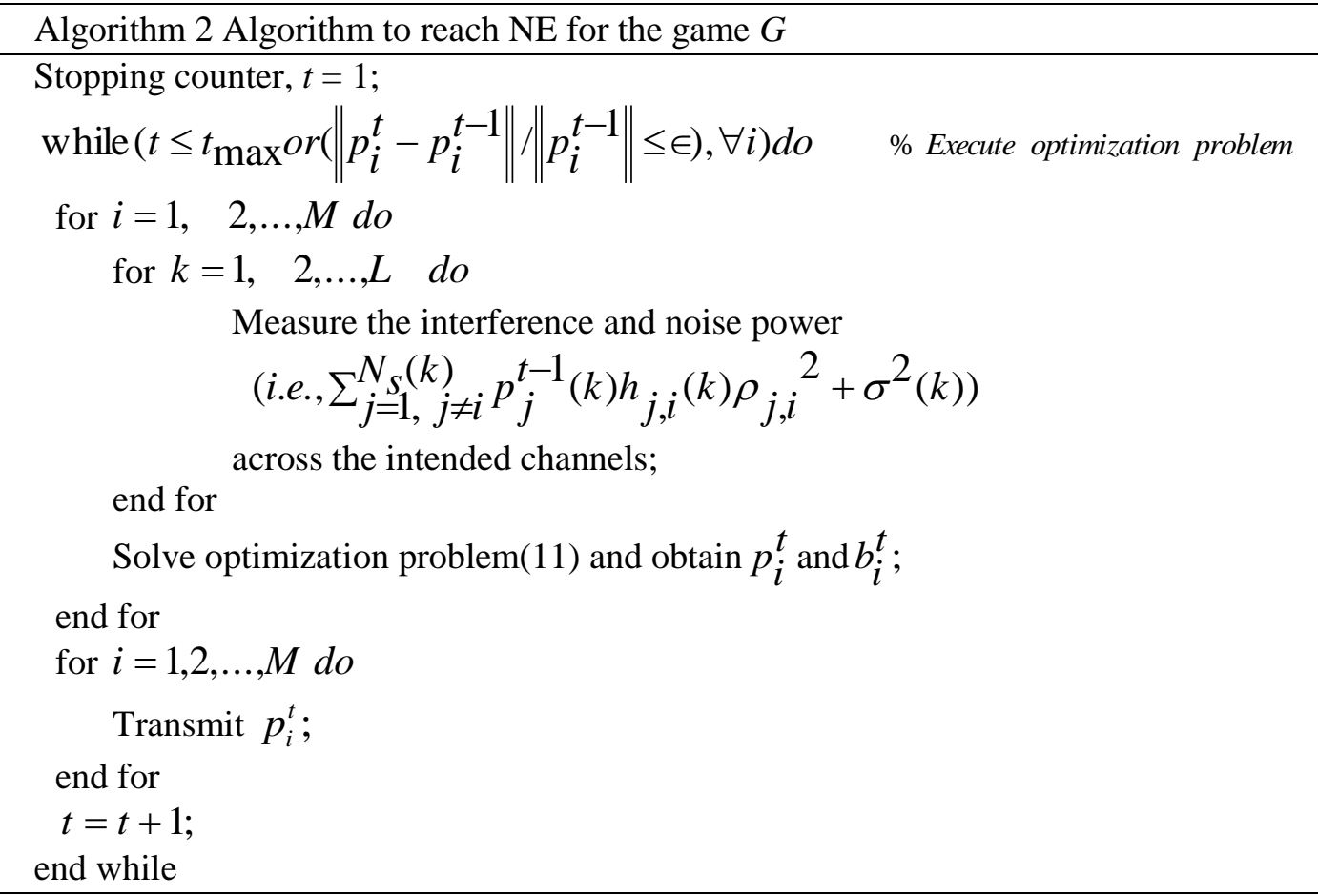

In the developed user-based distributed approach, each SU requires only local information- (i) possible number of users at next time instant $N_{s}(k), \forall k$ and measurement of interference and noise power.

\section{Find Power Efficiency and Net Transmission Cost for each usage Pattern Across Channels.}

\section{Power efficiency and Net transmission cost:}

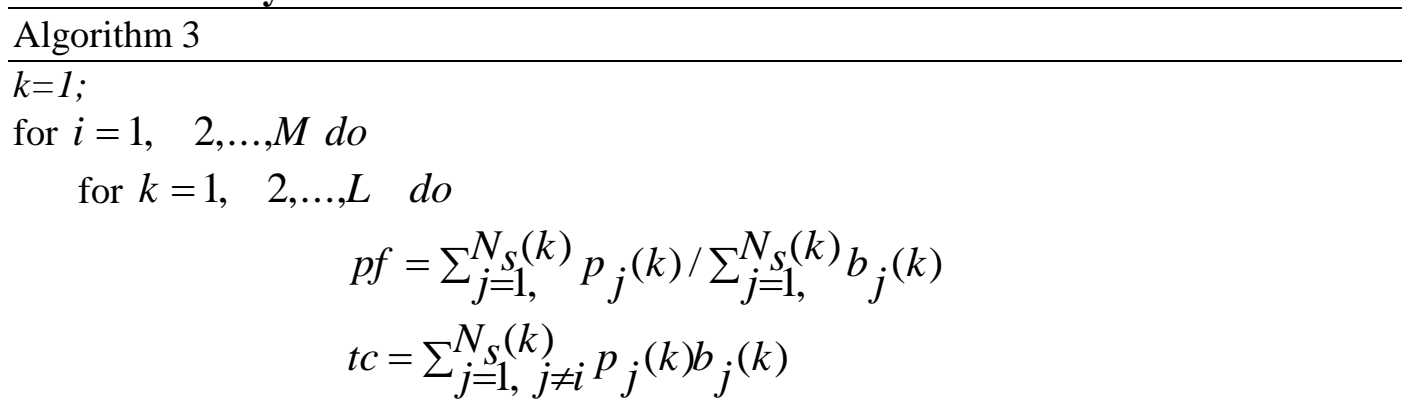
end for

end for

\section{Simulation and Analytical Results}

In cellular system leaders are base stations (BS) and followers are cognitive radio networks (CRN). In the simulations, we assume that there are $L=11$ available channels and a total of $M=10$ secondary users. Ten usage patterns across channels are applied for each of three Modulation schemes QAM, ASK and PSK. Table 7 provides information on the channel quality for all $L$ channels. Table 8 lists the minimum rate requirement for each 
SU [4]. Finally, Table 9 contains all other system parameters that are relevant to our resource allocation framework.

\section{Table 2. Usage Pattern across Channels}

Case 1: All users of all channels used:

\begin{tabular}{c|l|l|l|l|l|l|l|l|l|l|l}
\hline $\begin{array}{c}\text { Channel, } \\
K\end{array}$ & 1 & 2 & 3 & 4 & 5 & 6 & 7 & 8 & 9 & 10 & 11 \\
\hline \hline User, 1 & 1 & 1 & 1 & 1 & 1 & 1 & 1 & 1 & 1 & 1 & 1 \\
\hline User, 2 & 1 & 1 & 1 & 1 & 1 & 1 & 1 & 1 & 1 & 1 & 1 \\
\hline User, 3 & 1 & 1 & 1 & 1 & 1 & 1 & 1 & 1 & 1 & 1 & 1 \\
\hline User, 4 & 1 & 1 & 1 & 1 & 1 & 1 & 1 & 1 & 1 & 1 & 1 \\
\hline User, 5 & 1 & 1 & 1 & 1 & 1 & 1 & 1 & 1 & 1 & 1 & 1 \\
\hline User, 6 & 1 & 1 & 1 & 1 & 1 & 1 & 1 & 1 & 1 & 1 & 1 \\
\hline User, 7 & 1 & 1 & 1 & 1 & 1 & 1 & 1 & 1 & 1 & 1 & 1 \\
\hline User, 8 & 1 & 1 & 1 & 1 & 1 & 1 & 1 & 1 & 1 & 1 & 1 \\
\hline User, 9 & 1 & 1 & 1 & 1 & 1 & 1 & 1 & 1 & 1 & 1 & 1 \\
\hline User, 10 & 1 & 1 & 1 & 1 & 1 & 1 & 1 & 1 & 1 & 1 & 1 \\
\hline \hline
\end{tabular}

Table 3. SINR( All Channels of all users are used)

\begin{tabular}{|l|l|l|l|l|l|l|l|l|l|l|}
\hline 0.00064 & 0.00097 & 0.00062 & 0.00113 & 0.00089 & 0.00023 & 0.00061 & 0.00096 & 0.00085 & 0.00089 & 0.00068 \\
\hline 0.00048 & 0.00095 & 0.00082 & 0.00083 & 0.00131 & 0.00061 & 0.00053 & 0.00064 & 0.00047 & 0.00075 & 0.00090 \\
\hline 0.00071 & 0.00075 & 0.00104 & 0.00250 & 0.00080 & 0.00055 & 0.00072 & 0.00097 & 0.00073 & 0.00072 & 0.00067 \\
\hline 0.00065 & 0.00064 & 0.00067 & 0.00179 & 0.00138 & 0.00044 & 0.00115 & 0.00090 & 0.00100 & 0.00063 & 0.00060 \\
\hline 0.00043 & 0.00077 & 0.00066 & 0.00077 & 0.00105 & 0.00048 & 0.00068 & 0.00096 & 0.00035 & 0.00066 & 0.00059 \\
\hline 0.00054 & 0.00082 & 0.00079 & 0.00149 & 0.00158 & 0.00083 & 0.00114 & 0.00065 & 0.00040 & 0.00102 & 0.00066 \\
\hline 0.00036 & 0.00117 & 0.00078 & 0.00122 & 0.00121 & 0.00054 & 0.00075 & 0.00049 & 0.00052 & 0.00088 & 0.00035 \\
\hline 0.00052 & 0.00041 & 0.00108 & 0.00128 & 0.00142 & 0.00019 & 0.00110 & 0.00082 & 0.00040 & 0.00070 & 0.00052 \\
\hline 0.00036 & 0.00094 & 0.00087 & 0.00082 & 0.00124 & 0.00052 & 0.00042 & 0.00081 & 0.00083 & 0.00072 & 0.00041 \\
\hline 0.00045 & 0.00070 & 0.00065 & 0.00180 & 0.00094 & 0.00030 & 0.00098 & 0.00061 & 0.00053 & 0.00092 & 0.00039 \\
\hline
\end{tabular}

We select channels in Table below of high SINR which is in Table 3. We can apply several thresholds and can find cases.

Table 4. Threshold of SINR

\begin{tabular}{|c|c|c|}
\hline Case & $\begin{array}{c}\text { Threshold of } \\
\text { SINR }\end{array}$ & $\begin{array}{c}\text { Total no of channel occupied by } \\
\text { user }\end{array}$ \\
\hline 1 & 0.0000 & 110 \\
\hline 2 & 0.0003 & 107 \\
\hline 3 & 0.0004 & 102 \\
\hline 4 & 0.0005 & 90 \\
\hline 5 & 0.0006 & 80 \\
\hline 6 & 0.0007 & 60 \\
\hline 7 & 0.0008 & 47 \\
\hline
\end{tabular}

When the product of channel power and channel gain $\mathrm{H}$ increases, the SINR increases. On the other hand when the summation of interference and channel noise increases, the SINR decreases. It is not logical to allocate power to poorer channel SNIR. Hence we discard the channel. 
Table 5. Usage Pattern Across Channels

Case 3:

\begin{tabular}{c|c|c|c|c|c|c|c|c|c|c|c}
\hline $\begin{array}{c}\text { Channel, } \\
K\end{array}$ & 1 & 2 & 3 & 4 & 5 & 6 & 7 & 8 & 9 & 10 & 11 \\
\hline User, 1 & 1 & 1 & 1 & 1 & 1 & 0 & 1 & 1 & 1 & 1 & 1 \\
\hline User, 2 & 1 & 1 & 1 & 1 & 1 & 1 & 1 & 1 & 1 & 1 & 1 \\
\hline User, 3 & 1 & 1 & 1 & 1 & 1 & 1 & 1 & 1 & 1 & 1 & 1 \\
\hline User, 4 & 1 & 1 & 1 & 1 & 1 & 1 & 1 & 1 & 1 & 1 & 1 \\
\hline User, 5 & 1 & 1 & 1 & 1 & 1 & 1 & 1 & 1 & 0 & 1 & 1 \\
\hline User, 6 & 1 & 1 & 1 & 1 & 1 & 1 & 1 & 1 & 1 & 1 & 1 \\
\hline User, 7 & 0 & 1 & 1 & 1 & 1 & 1 & 1 & 1 & 1 & 1 & 0 \\
\hline User, 8 & 1 & 1 & 1 & 1 & 1 & 0 & 1 & 1 & 1 & 1 & 1 \\
\hline User, 9 & 0 & 1 & 1 & 1 & 1 & 1 & 1 & 1 & 1 & 1 & 1 \\
\hline User, 10 & 1 & 1 & 1 & 1 & 1 & 0 & 1 & 1 & 1 & 1 & 0 \\
\hline \hline
\end{tabular}

Remaining three methods are triple channel selection, double channel selection and single channel selection. In these methods such as single channel selection system power saves because weaker channels get no power. In our multiuser multichannel system only single channel with maximum SINR gets power for each user in single channel selection.

\section{Table 6. Usage Pattern across Channels}

Case 10: Single channel selection

\begin{tabular}{c|c|c|c|c|c|c|c|c|c|c|c}
\hline $\begin{array}{c}\text { Channel, } \\
K\end{array}$ & 1 & 2 & 3 & 4 & 5 & 6 & 7 & 8 & 9 & 10 & 11 \\
\hline \hline User, 1 & 0 & 0 & 0 & 1 & 0 & 0 & 0 & 0 & 0 & 0 & 0 \\
\hline User, 2 & 0 & 0 & 0 & 0 & 1 & 0 & 0 & 0 & 0 & 0 & 1 \\
\hline User, 3 & 1 & 0 & 0 & 1 & 0 & 0 & 0 & 1 & 0 & 0 & 0 \\
\hline User, 4 & 0 & 0 & 0 & 1 & 0 & 0 & 1 & 0 & 1 & 0 & 0 \\
\hline User, 5 & 0 & 0 & 0 & 0 & 1 & 0 & 0 & 0 & 0 & 0 & 0 \\
\hline User, 6 & 0 & 0 & 0 & 0 & 1 & 1 & 0 & 0 & 0 & 1 & 0 \\
\hline User, 7 & 0 & 1 & 0 & 1 & 0 & 0 & 0 & 0 & 0 & 0 & 0 \\
\hline User, 8 & 0 & 0 & 1 & 0 & 1 & 0 & 0 & 0 & 0 & 0 & 0 \\
\hline User, 9 & 0 & 0 & 0 & 0 & 1 & 0 & 0 & 0 & 0 & 0 & 0 \\
\hline User, 10 & 0 & 0 & 0 & 1 & 0 & 0 & 0 & 0 & 0 & 0 & 0 \\
\hline \hline
\end{tabular}

Only two channels with maximum two SINR gets power for each user in double channel selection. Triple channel selection is as so.

Table 7. Channel Quality Parameters

\begin{tabular}{c|c|c|c|c|c|c|c|c|c|c|c}
\hline \hline Channel, $K$ & 1 & 2 & 3 & 4 & 5 & 6 & 7 & 8 & 9 & 10 & 11 \\
\hline \hline$\sigma^{2}(k),\left(\times 10^{-3}\right)$ & 5 & 4 & 3 & 2 & 2.5 & 6 & 4 & 4 & 5 & 3.5 & 4.5 \\
\hline
\end{tabular}

We study the performance of Stackelberg game of cognitive radio network. In the simulations, we assume that there are $L=11$ available channels and a total of $M=10$ secondary users. 


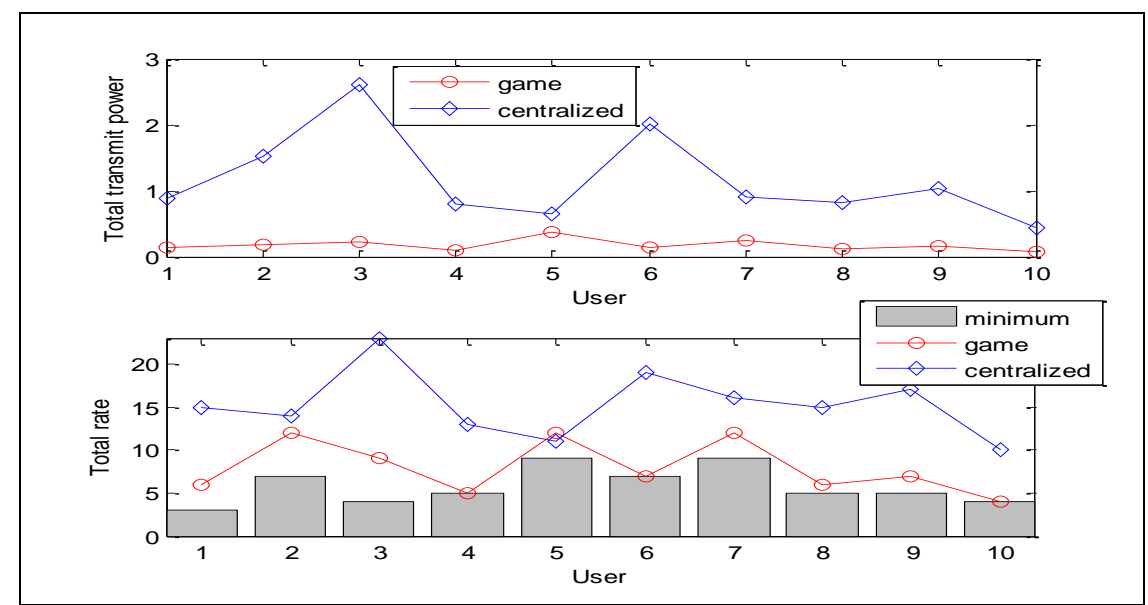

Figure 5.1. Allocation of (a) Total Transmit Power and (b) Total Rate of CRs for QAM as in Case 6

Figure 5.1(a) shows the allocation of total transmit power across users from both centralized (Eq.(4)) and distributed (Eq.(11) )schemes, respectively. Figure 5.1(b) shows the allocation of total rate across users from both centralized (Eq.(4)) and distributed (Eq.(11)) schemes respectively. We see from Figures 5.1 that both total allocated power and rate across users in distributed case are comparable to centralized scheme. Because in the game (Eq.(11)) maximum limit on power is set as the minimum of maximum usual limit on power and the upper bound. The upper bound can be obtained from dividing interference temperature threshold by the product of channel power gain at some location and possible number of users at next time instant as explained in (Eq.(13)) and (Eq.(14)). For the above approximations distributed solution gives less power than the counterpart. Additionally, our proposed distributed resource allocation scheme is successful in meeting minimum rate requirements for all SUs. The reason is obvious from the proposed userbased optimization problem formulation (Eq.(11)) after checking the feasibility of the optimization problem solution. The feasibility is determined by user minimum rate requirement (constraint (CG3). For each user, if the optimization problem is feasible, the distributed scheme is guaranteed to be successful in meeting the rate requirements for all SUs. Figure 5.1(b) clearly indicates that the proposed game statistics ensure the minimum rate requirement for all users.

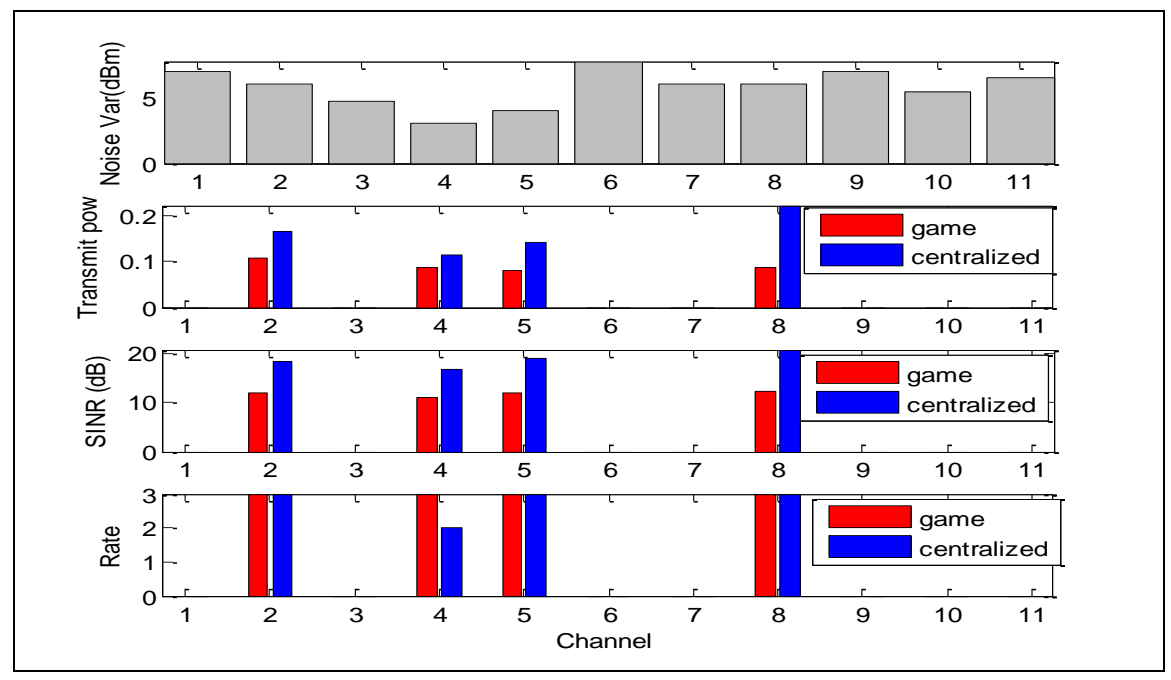

Figure 5.2. Allocation of Transmit Power and Rate with Channel Noise Variance and SINR for $5^{\text {th }}$ user of $5^{\text {th }}$ Scenario as in Case 6 of QAM 
In Figure 5.2. $1^{\text {st }}, 3^{\text {rd }}, 6^{\text {th }}, 7^{\text {t }}, 9^{\text {th }}, 10^{\text {th }}$ and $11^{\text {th }}$ channel is null (allocated power is 0 ) as in Case 6. Because these three channels have higher interference and noise and also moderate power and channel gain product. Because SINR of these channel are less than threshold.

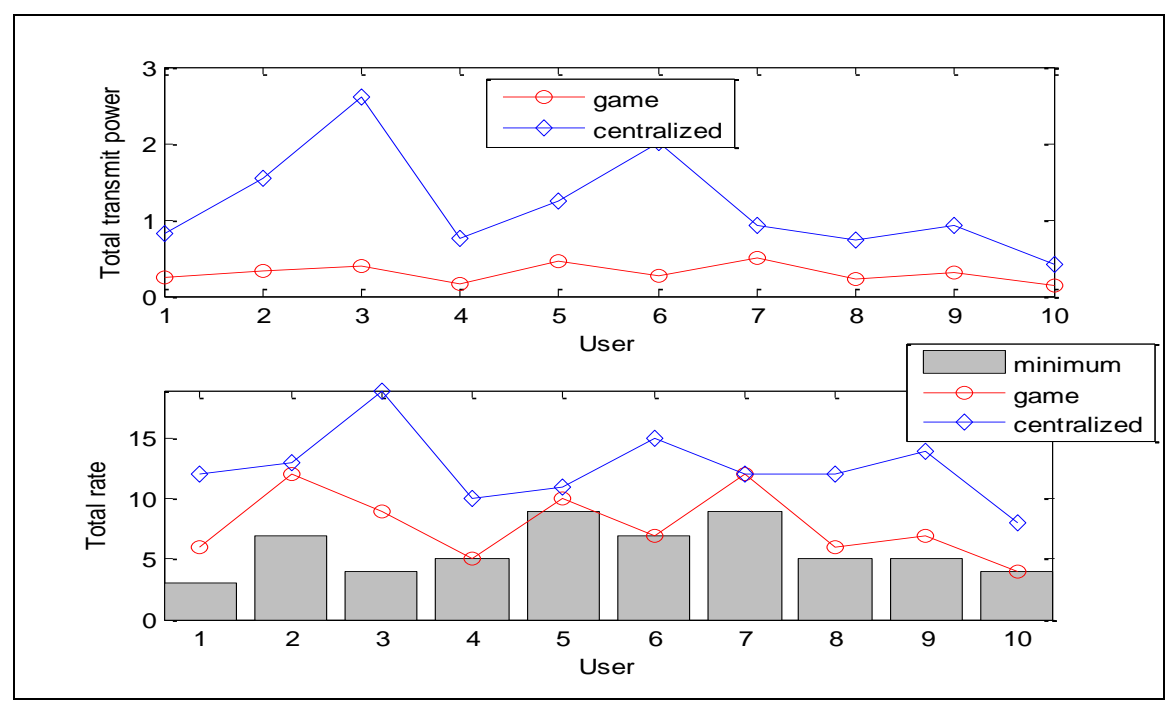

Figure 5.3. Allocation of Total Transmit Power and Total Rate of CRs for
ASK as in Case 6

Figure 5.3. shows for a certain bit error rate $\mathrm{C}_{\text {qarg }}$ is larger in QAM than in ASK and PSK. So QAM needs more SINR. If more number of channels are vacant then busy channels get higher value of power. Receiver design of CDMA system is more complex for more number of busy(occupied) channels. If total power each user is increased then bit rate increased and vice versa. For maximum number of channel occupied the difference between power of centralized and distributed scheme is large. This is also true for rate. For minimum number of channel occupied the difference between power of centralized and distributed scheme is small. This is also true for rate. For same number of channels utilization game theory require less power than centralized scheme. Consequently, rates become less in game theory than centralized scheme. Sacrificing lower rates of user do not violate the minimum threshold rates.

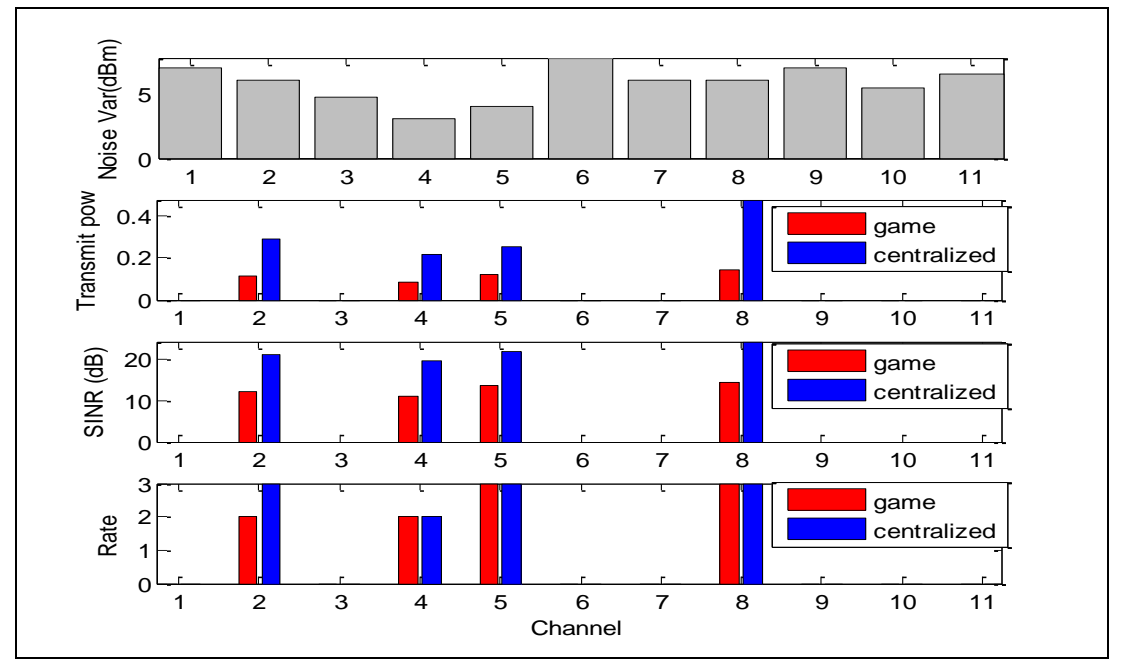

Figure 5.4. Allocation of Transmit Power and Rate with Channel Noise Variance and SINR for $5^{\text {th }}$ user of Case 6 of ASK 
In Figure 5.4. ${ }^{\text {st }}, 3^{\text {rd }}, 6^{\text {th }}, 7^{\text {th }} 9^{\text {th }}, 10^{\text {th }}$ and $11^{\text {th }}$ channel is null (allocated power is 0 ) as in Table 5.7. Because these channel have higher interference and noise and also moderate power and channel gain product. These two causes lesser SINR which nullify the channel parameters (Transmit power, SINR, Rate). Because SINR of these channel are less than threshold.

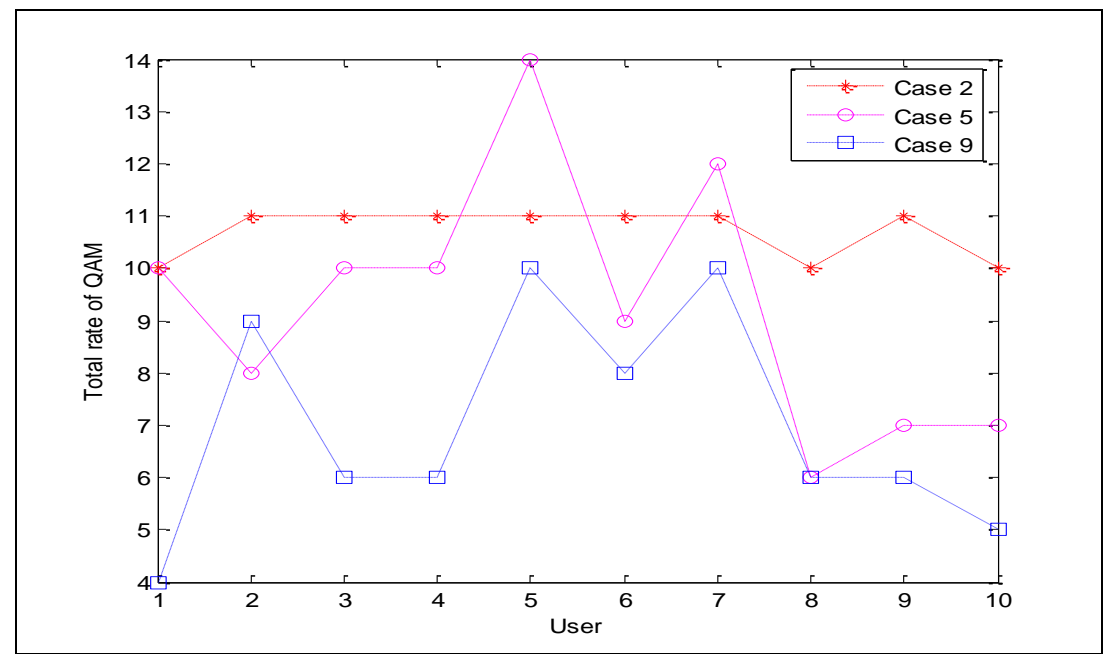

Figure 5.5. Allocation of Rate of Case 2, Case 5 and Case 9 for QAM

For all cases of QAM our program successfully give result. Figure 5.5. we show case 2, case 5 and case 9 only.

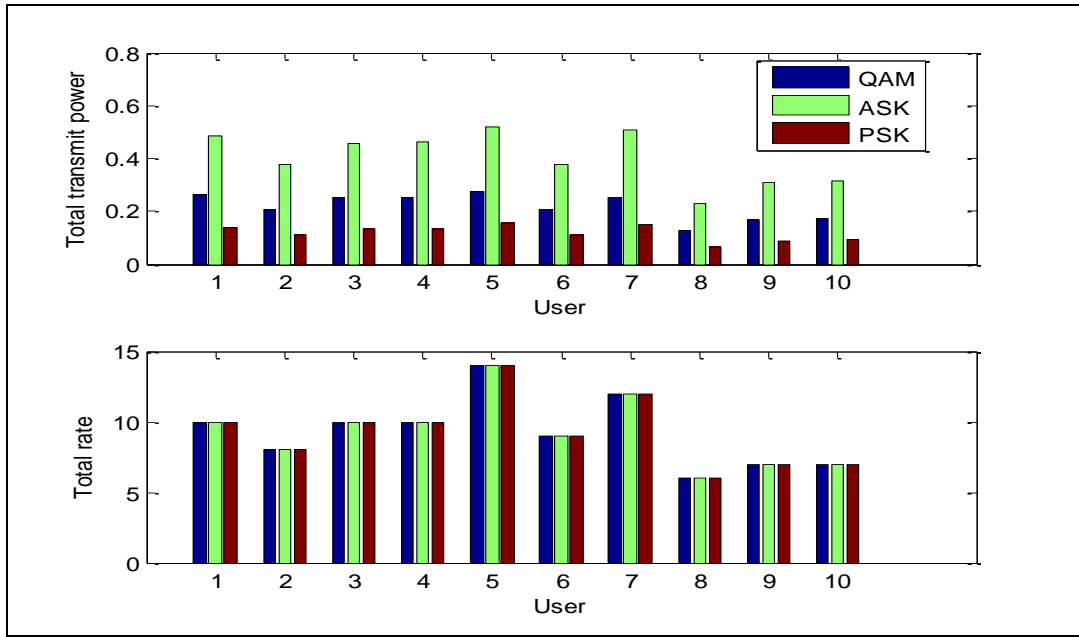

Figure 5.6. Comparison of Total Transmit Power and Total Rate for Game Theory of CRs for QAM, ASK and PSK Respectively as Case 5

Figure 5.6. presents the of total transmit power across users of distributed scheme for QAM, ASK and PSK modulation. From the constant $C_{\text {qarg }}$ calculated for QAM, ASK and PSK we can predict that the PSK has the lowest power allocation and the ASK has the highest power allocation. QAM needs moderate power as it has two components.

Table 7 provides information on the channel quality for all $L$ channels. Table 8 lists the minimum rate requirement for each SU [4]. Finally, Table 9 contains all other system parameters that are relevant to our resource allocation framework. Based on all this information, our objective is to find the optimal transmit power and rate that each of the 
$M$ SUs should employ to guarantee their QoS(BER) through our user-based distributed approach.

Table 8. Minimum Rate Requirements of Users

\begin{tabular}{c|c|c|c|c|c|c|c|c|c|c}
\hline \hline User, $i$ & 1 & 2 & 3 & 4 & 5 & 6 & 7 & 8 & 9 & 10 \\
\hline$R_{i}^{l}$ & 3 & 8 & 4 & 12 & 9 & 7 & 14 & 5 & 10 & 8 \\
\hline \hline
\end{tabular}

Table 9. System Parameters

\begin{tabular}{c|c}
\hline$\Pi_{i}^{\max } \forall i$ & 1.0 \\
\hline$p_{i}^{\max }(k) \forall i, k$ & 5 \\
\hline$b_{i}^{\max }(k) \forall i, k$ & 6 \\
\hline$p_{e, i}^{t h} \forall i$ & $10^{-3}$ \\
\hline$I_{t h}(k) \forall k$ & $200 \times \sigma^{2}(k)$ \\
\hline$R_{c h}^{u}(k) \forall k$ & 20 \\
\hline$p_{j, i}$ & 0.03125 \\
\hline${ }^{t}{ }_{\max }$ & 10 \\
\hline$\varepsilon$ & 0.01 \\
\hline \hline
\end{tabular}

Figure 1(a) shows the allocation of total transmit power across users from both centralized (Eq.(7) )and distributed schemes, respectively. Figure 1(b) shows the allocation of total rate across users from both centralized (Eq.(7)) and distributed schemes, respectively. We see from Figures 1(a) and 1(b) that both total allocated power and rate across users in distributed case are comparable to centralized scheme. Because in the game (11) maximum limit on power is set as the minimum of maximum usual limit on power and the upper bound obtained from dividing interference temperature threshold by the product of channel power gain at some location and possible number of users at next time instant. Similarly the upper bound on maximum limit on rate is set as the minimum of maximum usual limit on rate and the upper bound obtained from dividing the total rate supporting capability of a channel by possible number of users at next time instant. For the above approximations distributed solution gives less power and bit rate than the counterpart. Additionally, our proposed distributed resource allocation scheme is successful in meeting minimum rate requirements for all SUs. The reason is obvious from the proposed user-based optimization problem formulation (11) after checking the feasibility of the optimization problem solution. The feasibility is determined by user minimum rate requirement (constraint (CG3). For each user, if the optimization problem is feasible, the distributed scheme is guaranteed to be successful in meeting the rate requirements for all SUs.

Figure 2 presents the transmit power and rate allocation across channels for user 4 (assuming scalarization parameters $\tau_{1}$ and $\tau_{2}$ to 0.5 ) from the proposed distributed scheme along with centralized scheme (Eq.(7)). The channel noise variance and resulting SINR are also shown for reference. User 4 operates on channels 2, 3, 5, 6, 7, 9, 10 and 11. A game theory does not always give optimal result. We can observe the effect in the channel 5 and 6 . Here game theory requires more transmit power.

Figure 3(a) and 3(b) show the resulting total interference power and allocation of total rate, respectively, across channels from distributed scheme along with upper limits. We see from Figures 3(a) and 3(b) that both resulting total interference power and total rate 
across channels do not violate the corresponding upper limits. That is, the conservative approach based on constraints $C G 3$ and $C G 4$ in the proposed distributed case is successful in satisfying the system constraints $C 3$ and $C 4$.

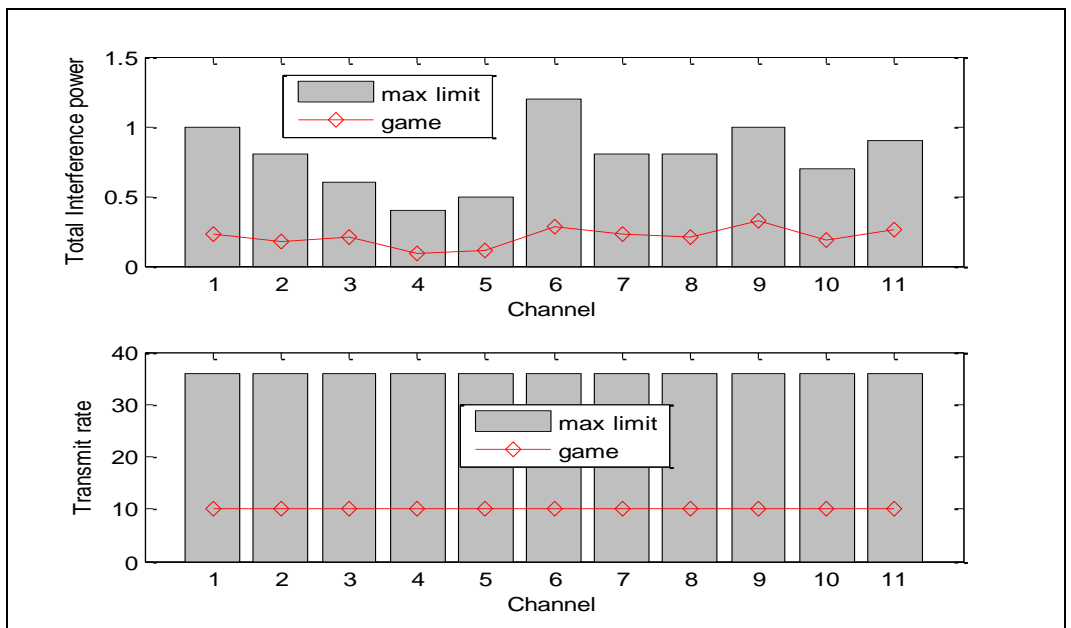

\section{Figure 5.6. Maximum Limit and Game Based Calculated Limit for QAM as Case 1}

In Case 1. all channel are active so sum of maximum power and rate allocated which do not exceed maximum limit.

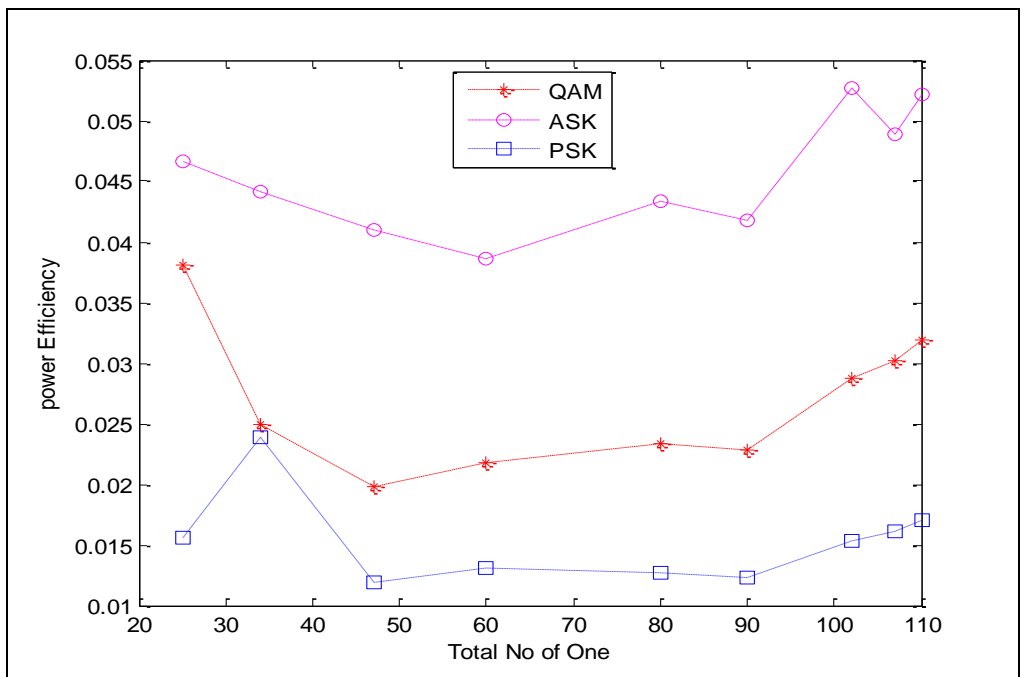

\section{Figure 5.7. Curves for Power Efficiency of each Usage Pattern across Channels Shown}

Figure 5.7. shows that less channel utilization more power is needed. Hence power efficiency greater. ASK requires maximum power and PSK requires minimum power. At moderate channel usage power efficiency is minimum.

Figure 5.8. shows net transmission cost(Eq.(3.2)) is lower for less and moderate channel usage. Here ASK requires maximum transmission cost and PSK requires minimum transmission cost. Though in maximum busy channels total power decreased but amount of increased transmission rate overcomes this. So Net transmission cost increased at maximum usage of channels. 


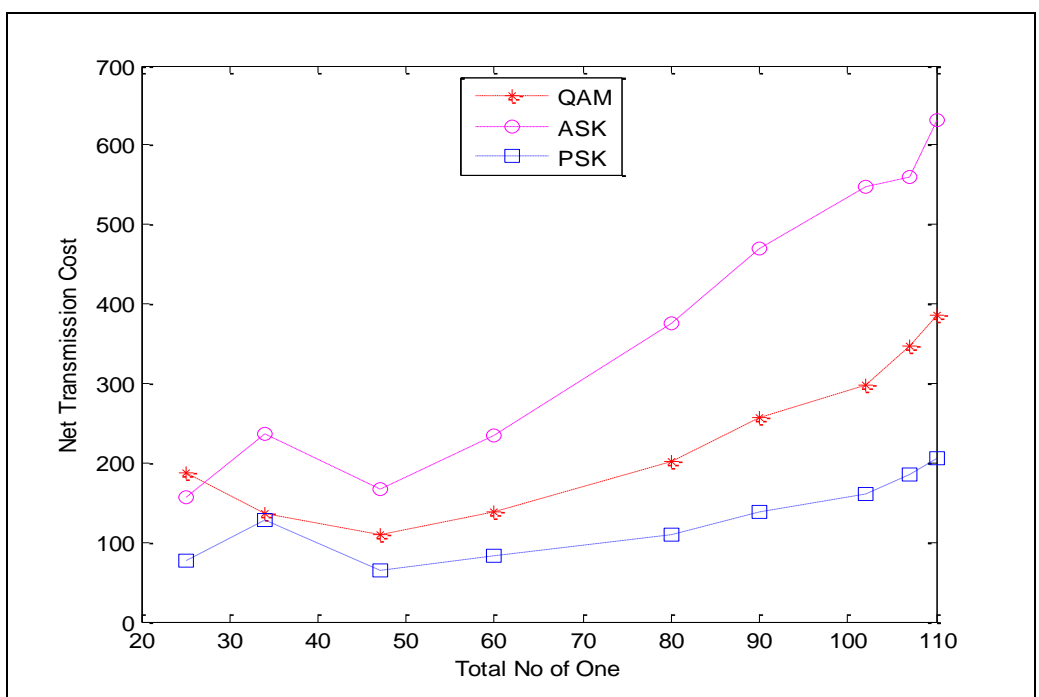

Figure 5.8. Net Transmission Cost of Each Usage Pattern across Channels Shown

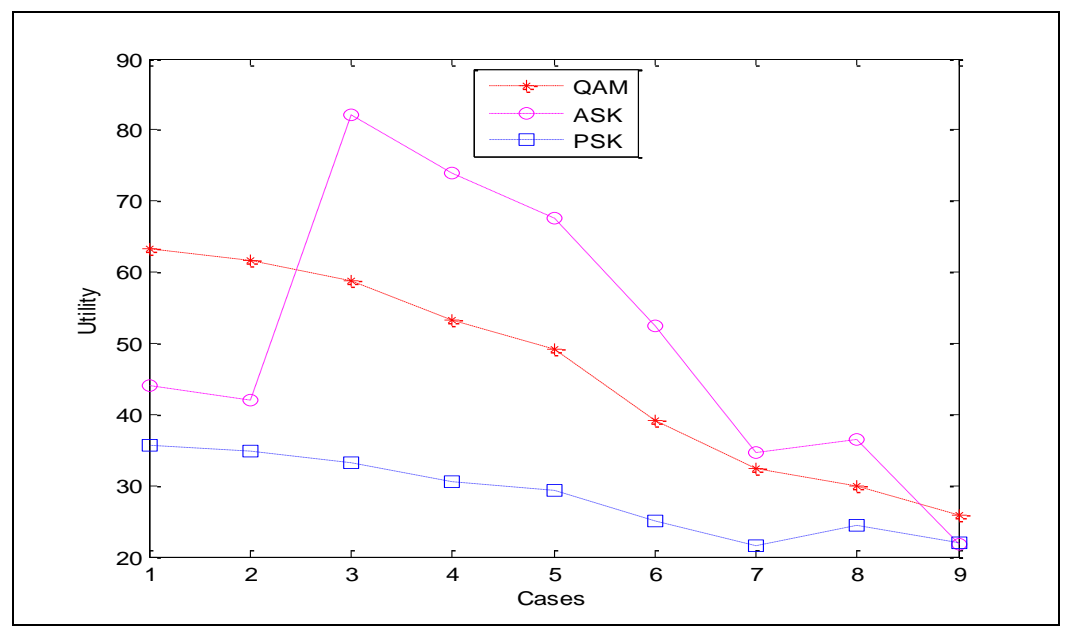

Figure 5.9. Utility of each Base Stations

Figure 5.9. shows if more channels are busy then the summation of secondary users increased from (Eq.(4.1) ). The utility of corresponding BS are decreased. Out of three modulation PSK requires hightest power then QAM and ASK. Hence Utility of each Base Station for ASK is maximum and PSK achieves minimum utility.

\section{Conclusion}

Now adays Game theory based distributed approach is used for its low complexity and easier implementability. In this paper, we consider the problem of power allocation to maximize utilities of users (BS and CRs) in a cognitive radio network that is suitable for Stackelberg Game. Using game theory, we propose a distributed power control scheme to protect the communications of primary users while providing maximum possible utilization of base stations and cognitive radios. We assume that multiple secondary users may coexist in a single channel and a single secondary user can simultaneously employ multiple channels to meet its rate requirements. 


\section{References}

[1] G. Scutari, D.P. Palomar, F. Facchinei and J.S. Pang, "Convex Optimization, Game Theory, and Variational Inequality Theory”, IEEE Signal Processing Magazine, vol. 27, no. 3, (2010), pp. 35-49.

[2] T. Zhang, W. Chen and Z. Z. Cao, "Hierarchic Power Allocation for Spectrum Sharing in OFDM-Based Cognitive Radio Networks", Vehicular Technology, IEEE Transaction, vol. 63, Issue 8, (2014).

[3] L. Aker and B Natarajan, "QoS Constrained Resource allocation to Secondary Users in Cognitive Radio Networks", Elsevier, Special Issue of Computer Communication on Cognitive Radio and Dynamic Spectrum Sharing Systems", (2009).

[4] M. Chaing. P. Hande, T. Lan and C. W. Tan, "Power Control in Wireless Cellular Networks", (2008).

[5] E. Hossain, L. Le, N. Devroye and M. Vu, "Cognitive Radio: From Theory to Practical Network Engineering", Springer, New Directions in Wireless Communications Research, (2009), pp. 251-289.

[6] L. Akter and B. Natarajan, "Game Theory based Distributed Approach for Power and Rate Allocation to Secondary Users in Cognitive Radio Networks", International Conference on Informatics, Electronics \& Vision (ICIEV)", (2013).

[7] A. B. MacKenzie and L A. DaSilva, "Game theory for wireless engineers", Morgan and Claypool Publishers.

[8] S. C. Choi, W. S. Dasarbo and P. T. Harker, "Product positioning under price competition", INFORMS. Management Science, vol. 36, no. 2, (1990), pp. 175-199.

[9] N. N. Omidvar and B. H. Khalaj, "A Game Theoretic Approach for Power Allocation in the Downlink of Cognitive Radio Networks", Computer Aided Modeling and Design of Communication Links and Networks (CDMAD)", 2011 IEEE.

[10] X. Kang, Y.-C. Liang and H. K. Garg, "Distributed Power control for Spectrum-Sharing Femtocell Networks using Stackelberg game", IEEE ICC, (2011).

[11] T. Marler, "A study of multiobjective optimization methods for engineering".

[12] V. U. Sankar and V. Sharma, "Pricing and Power allocation in Femtocells using Stackelberg Game Theory", IEEE SPCOM, (2014).

[13] M.O. Rahman and Asaduzzaman "Optimal Power Allocation for Multichannel Cognitive Radio Systems Using Stackelberg Game ”, 1st International Conference ICAICT, (2016).

\section{Authors}

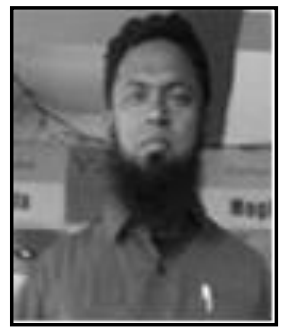

Mohammad Obaidur Rahman, he received the B. Sc. Engineering Degree in Electrical and Electronic Engineering from Bangladesh University of Engineering and Technology (BUET), Bangladesh, in 1998. He has currently received M. Engineering degree from Department of CSE, Chittagong University of Engineering and Technology (CUET) From September 2001 to onwards he has been serving as a faculty member in the Department of Computer Science and Engineering, CUET, Chittagong, Bangladesh. He is currently working toward the Ph.D. degree as a part-time basis in the Department of CSE, CUET, Bangladesh.

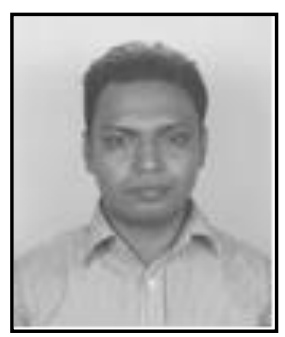

Asaduzzaman, he received the B. Sc. Engineering Degree in Electrical and Electronic Engineering from Chittagong University of Engineering and Technology(CUET), Bangladesh, in 2001. From 2001 to onwards has been serving as a faculty member in the Department of Computer Science and Engineering, CUET, Chittagong, Bangladesh. He received his Ph.D. from the Department of Electrical Engineering, University of Ulsan, Korea in 2010. His major research interests include wireless communication systems with emphasis on cooperative communications and MIMO systems, wireless sensor networks, modulation and coding techniques, cognitive radio, Internet of Things etc. 
International Journal of Advanced Science and Technology Vol.107 (2017) 